\title{
Intégrer la dimension genre dans l'enseignement supérieur : transformation de pratiques au niveau individuel et institutionnel
}

Annick Rossier, Jessica Dehler, Bernadette Charlier et Hélène Füger

\section{(2) OpenEdition}

Journals

Édition électronique

URL : http://journals.openedition.org/ripes/398

DOI : 10.4000/ripes.398

ISSN : 2076-8427

Éditeur

Association internationale de pédagogie universitaire

Référence électronique

Annick Rossier, Jessica Dehler, Bernadette Charlier et Hélène Füger, «Intégrer la dimension genre dans l'enseignement supérieur : transformation de pratiques au niveau individuel et institutionnel », Revue internationale de pédagogie de l'enseignement supérieur [En ligne], 26(2) | 2010, mis en ligne le 08 février 2011, consulté le 07 septembre 2020. URL : http://journals.openedition.org/ripes/398 ; DOI : https://doi.org/10.4000/ripes.398

Ce document a été généré automatiquement le 7 septembre 2020

Article L.111-1 du Code de la propriété intellectuelle. 


\title{
Intégrer la dimension genre dans l'enseignement supérieur : transformation de pratiques au niveau individuel et institutionnel
}

\author{
Annick Rossier, Jessica Dehler, Bernadette Charlier et Hélène Füger
}

\section{Introduction}

1 Le projet « e-qual : enseignement genre qualité » (ci-après, projet e-qual) a été mis en place conjointement par le Centre de Didactique Universitaire et par le Service de l'égalité de l'Université de Fribourg, avec le concours du Service de la gestion qualité et le soutien du Rectorat. Le projet avait pour objectif d'intégrer la dimension genre comme élément constitutif de l'assurance qualité dans la pratique, la formation et l'évaluation de l'enseignement supérieur, plus particulièrement à l'Université de Fribourg. Ainsi, dans le projet e-qual, la prise en compte de la dimension genre dans l'enseignement et le développement de sa qualité sont considérés comme des réponses pertinentes - bien qu'insuffisantes - au problème de la reproduction de la ségrégation horizontale (au moment des choix d'études, femmes et hommes se répartissent de manière asymétrique dans beaucoup de domaines) et de la ségrégation verticale (inégalités dans l'accès à la carrière professionnelle et académique en particulier). Plusieurs facteurs ont facilité la mise en place de ce projet à l'Université de Friboug: l'existence d'une préoccupation de la part du Rectorat, une habitude de collaboration entre les trois principaux services universitaires concernés par cette problématique et, finalement, le soutien du programme fédéral égalité des chances qui a largement contribué à financer le projet.

2 Le développement de la qualité de l'enseignement par la prise en compte de l'aspect genre comme facteur de cette qualité vise une transformation non seulement au niveau des pratiques des individus (enseignant-e-s), mais aussi au niveau institutionnel. 
L'enjeu du projet e-qual est de travailler à ces deux niveaux. Or, comment introduire un tel changement? Le succès des changements visés au sein d'une institution dépend, pour une part importante, de la manière dont ces derniers sont introduits (Giauque, Barbey, Duc, 2008). Ces auteurs relèvent en particulier l'importance d'un pilotage participatif : «Cette étude démontre qu'il n'existe pas de one best way des réformes et que le ressenti en termes de performance individuelle ou organisationnelle dépend bien plus d'éléments symboliques, de pilotage organisationnel, de démocratie participative, de création d'espace d'autonomie et de valeurs, que des principes et outils de gestion qui émargent de la philosophie managériale » (Giauque, Barbey et Duc, 2008, p 796). Dans cette perspective, le projet a pris le parti de faire participer autant le corps enseignant que les représentants institutionnels (Rectorat, décanat) de l'Université de Fribourg dans les différentes étapes et dans le processus d'élaboration des résultats du projet.

Le projet, d'une durée de 24 mois, s'est terminé en mars 2010. Il a été organisé selon plusieurs axes de travail, centrés sur le changement des pratiques individuelles et sur la prise en compte de ces préoccupations dans les dispositifs et structures institutionnelles.

\section{Sensibilisation au genre, de quoi parle-t-on?}

Le genre joue un rôle à l'université comme partout où des êtres humains ont des interactions sociales. Le genre se construit dans les interactions sociales et s'inscrit dans les normes et les structures formelles et informelles des institutions. Ainsi, ce concept se distingue du sexe car il se construit socialement par « doing gender » dans un contexte donné et est renouvelé dans les interactions (West \& Zimmerman, 1987). A l'université, l'enseignement est un des principaux espaces où les étudiant-e-s sont socialisé-e-s en ce qui concerne la recherche et leur domaine d'étude. La différence de genre risque d'être (re-)construite durant ces moments de formation si l'enseignant-e ne prend pas en compte cet aspect construit du genre. C'est le cas lorsque l'enseignement n'assure pas autant de chance d'apprentissage aux femmes qu'aux hommes. Voici quelques exemples où le concept de genre joue un rôle sur la qualité de l'enseignement: le sexe d'un-e étudiant-e peut influencer les attentes d'un-e enseignant-e concernant ses compétences, intérêts, potentiels; les références bibliographiques d'un cours montrent quel-le-s auteur-e-s sont considéré-e-s important-e-s dans le domaine; un langage qui n'est pas épicène s'adresse plus aux étudiants qu'aux étudiantes (Deaux \& Major, 1987 ; Gygax, Gabriel, Sarrasin, Oakhill, \& Garnham, 2008). Un enseignement sensible au genre tient compte du genre en étant attentif aux processus du "doing gender", en montrant la variété plutôt que les différences (présumées ou réelles), en réfléchissant aux mécanismes produisant des stéréotypes et aux inégalités de genre et en remettant en question les attributions marquant le masculin ou le féminin. Un enseignement de ce type demande de nombreuses compétences et une flexibilité importante (assurance et autoréflexion) de la part des enseignant-e-s. La sensibilisation au genre concerne toute démarche visant à rendre attentifs, conscients les individus quant au rôle qu'ils ont à jouer pour favoriser l'égalité des chances entre femmes et hommes. 


\section{Soutenir le changement des pratiques individuelles}

5 Le corps enseignant de l'Université de Fribourg a été impliqué dans la mise en œuvre du projet e-qual par l'intermédiaire du dispositif de formation continue Did@cTIC, proposé par le Centre de Didactique Universitaire. Une brève présentation du dispositif permet de mieux appréhender les changements introduits par le projet e-qual qui avait pour objectif de soutenir un changement de pratique au niveau individuel, tant chez des enseignant-e-s intervenant dans un module de formation que chez les participante-s. Les personnes intéressées ont ainsi pu être sensibilisées à la question du genre et ont évolué dans leur pratique professionnelle d'enseignement supérieur. Toutefois, ces pratiques d'enseignement sensibles au genre ne sont pas (encore) généralisées à l'ensemble du corps enseignant.

\subsection{Dispositif Did@cTIC}

$6 \quad$ Le Centre de Didactique Universitaire soutient depuis plus de sept ans la formation des enseignant-e-s de l'Université de Fribourg. Un ensemble de modules de formation aborde les questions essentielles de la pratique professionnelle en enseignement supérieur. Ce dispositif de développement professionnel des enseignant-e-s a été construit et régulé sur la base d'un ensemble d'analyses de besoins menées en 2002, 2005 et 2008. Ces analyses ont orienté la planification de l'offre du Centre de didactique, en particulier en distinguant les activités proposées aux assistant-e-s de celles destinées au corps professoral. La formation modulaire Did@cTIC s'adresse plus particulièrement aux enseignant-e-s faisant partie du corps des collaborateurs et collaboratrices scientifiques. Les modules proposés permettent une formation à la carte selon les besoins individuels. La formation peut être validée par un certificat (15 ECTS) ou un diplôme (30 ECTS).

7 L'offre destinée au corps professoral répond à des attentes plus spécifiques et ponctuelles. Elle correspond à un accompagnement individualisé et l'opportunité de participer à des communautés de pratique thématisées selon les attentes des professeur-e-s. Ces thèmes ont été identifiés lors d'entretiens auprès d'une trentaine de professeur-e-s.

8 Toute action de formation proposée par le Centre de didactique est guidée par les principes suivants, la formation est :

- Centrée sur la réalisation d'un projet individuel ;

- En lien avec la pratique en recourant aux projets personnels, aux communautés de pratique ;

- Flexible (certificat, diplôme, modules "à la carte", suivi individuel, communautés de pratique) ;

- Ouverte (intervention d'expert-e-s externes, accès à d'autres programmes) ;

- Bilingue ;

- Soutenue par l'usage des TIC ;

- Régulée de manière continue (évaluation de l'enseignement et analyses de besoins).

Les modules d'enseignement du dispositif Did@cTIC sont organisés en trois axes :

1. enseignement - apprentissage ;

2. évaluation ;

3. développement professionnel. 
Dans le cadre du projet e-qual, le dispositif Did@cTIC s'est enrichi de la prise en compte de la dimension genre dans chacun de ces axes. Elle s'est faite de deux manières : d'une part, l'offre de formation existante a été examinée, puis adaptée par le biais d'un conseil offert aux enseignant-e-s responsables et, d'autre part, quatre nouveaux modules tenant compte de la dimension genre ont complété l'offre existante.

\subsubsection{Adaptation des modules existants}

Le conseil aux enseignant-e-s responsables des modules existants du dispositif Did@cTIC a été abordé de deux manières. D'une part, tou-te-s les enseignant-e-s ont été contacté-e-s et informé-e-s de la possibilité d'être conseillé-e d'un point de vue genre. D'autre part, la collaboratrice scientifique du projet a pris contact de façon ciblée avec un certain nombre d'enseignant-e-s en leur expliquant pourquoi elle pensait que leur module se prêtait particulièrement bien à un tel conseil. Cette deuxième démarche $\mathrm{a}$ reçu un accueil favorable.

e conseil était basé sur une série de critères définis suite à l'analyse de la littérature concernant l'enseignement sensible au genre. Ces critères ont également été exploités lors de la conception d'une grille d'observation et d'auto-évaluation présentée plus loin dans ce texte.

13 Ainsi, durant la session 2008-2009 de la formation Did@cTIC, différents modules du dispositif ont été observés en tenant compte de quatre dimensions déterminant un enseignement sensible au genre, à savoir :

1. Assurer autant aux étudiantes qu'aux étudiants des opportunités d'apprentissage ;

2. Evaluer la sensibilité au genre de l'enseignement ;

3. Fournir un modèle d'enseignement sensible au genre « role model » ;

4. Sensibiliser les apprenant-e-s à la problématique des inégalités et du genre.

Ces observations ont été suivies d'un entretien individuel avec les formatrices de chaque module de base. L'échange a porté sur :

- Les ressources pédagogiques utilisées :

- $\circ$ Utilisation d'un langage épicène - dont la forme ne varie pas selon le genre - dans le discours oral et les textes écrits ;

- Choix d'exemples et d'images évitant de renforcer des stéréotypes de genre ;

- Destinataire principal des textes mis à disposition : les textes s'adressent-ils spécifiquement aux hommes, aux femmes ou aux deux genres?

- Les méthodes didactiques :

- ○Interactions avec les étudiant-e-s ;

- Modalités d'enseignement, par exemple, le travail en groupe suggérant la mixité des groupes.

Ce processus d'observation suivi d'un feedback a permis, d'une part, de sensibiliser les formatrices à la perspective genre et, d'autre part, d'apporter des pistes concrètes d'amélioration des modules de base proposés dans le dispositif Did@cTIC. Les conditions de réussite de ce type de sensibilisation se situent au niveau des formateurs et formatrices donnant les modules. En effet, la démarche est volontaire, il est nécessaire que chacun-e accepte d'être observé-e et d'avoir un entretien concernant la question du genre. Pour un échange constructif menant à des changements de pratique, une relation de confiance est nécessaire entre l'observateur ou l'observatrice 
et l'enseignant-e. L'enjeu de la réussite d'un changement individuel se joue dans une large mesure à ce niveau relationnel.

Cette approche de sensibilisation au genre peut être définie comme une approche participative. En effet, la démarche est entreprise uniquement si les formateurs et formatrices sont partis prenantes. De plus, les suggestions fournies lors du feedback doivent être reconnues par le formateur ou la formatrice comme un moyen efficace d'améliorer sa pratique pour qu'elles soient mises en oeuvre. Comme l'ont relevé Giauque, Barbey et Duc (2008), le facteur humain est essentiel dans le développement organisationnel et le rôle participatif des acteurs impliqués est une caractéristique favorisant le changement.

17 A titre d'illustration, voici comment s'est déroulée la démarche de conseil pour un module d'introduction à la didactique universitaire. Avant cette formation, les ressources (notes de cours, présentations multimédias) utilisées durant le cours ont été analysées. Quelques exemples mettant en scène des femmes ont été ajoutés. Le langage épicène a été appliqué partout. Ensuite, les activités ont été observées et le feedback détaillé a permis de souligner certaines pratiques pouvant être ajustées. Ainsi, par exemple, la composition des groupes lors d'une activité aurait pu être plus équilibrée. Enfin, une question a été ajoutée au questionnaire d'évaluation adressé aux étudiant-es permettant de se rendre compte d'une augmentation de la sensibilité aux genres se marquant particulièrement par le souci d'employer le langage épicène. Plus largement, ces changements de pratiques ont pu être transférés à d'autres enseignements pris en charge par la formatrice.

\subsubsection{Introduction de nouveaux modules}

18 La qualité de l'enseignement a été favorisée non seulement par la régulation, d'un point de vue genre, des modules de formation, mais également par la mise sur pied de quatre nouveaux modules à option. Le choix de ces modules a été déterminé par la pertinence de la prise en compte de la question du genre au regard de la thématique pédagogique abordée.

Des quatre modules, l'un concernait la question du genre de manière directe - Prendre en compte l'aspect genre dans son enseignement. Ce module traitait, par exemple, des approches théoriques du genre, du langage épicène et de l'évaluation de la sensibilité au genre d'un enseignement. Les autres modules abordaient la question de genre de manière transversale, leur thématique centrale étant focalisée sur la pédagogie universitaire. Dans le module - L'apprentissage collaboratif: comment créer des interactions qui stimulent l'apprentissage? - l'aspect genre était abordé avec des questions comme la distribution des rôles dans un groupe qui est souvent stéréotypée (femme pour rédiger le protocole; homme pour une présentation). Le module - Le processus d'évaluation perception, description, jugement - traitait les biais potentiels qui risquent de limiter la qualité d'évaluation des apprentissages. Le genre servait à illustrer certains biais comme, par exemple, les stéréotypes sur les aptitudes des femmes et des hommes. Les participant-e-s du module - Transfert de résultats de recherche dans sa pratique d'enseignement - ont élaboré des manières d'appliquer les résultats de la recherche genre dans leur enseignement.

En synthèse, l'expérience vécue dans le cadre du projet e-qual a incité le Centre de Didactique Universitaire à réguler son dispositif de formation en intégrant la question 
du genre au sein de modules abordant des thématiques de pédagogie universitaire. Cette approche permet de sensibiliser les enseignant-e-s à la question du genre en l'abordant de façon transversale et en la mettant en oeuvre de manière pratique dans la formation. Le dispositif Did@cTIC s'est ainsi enrichi - par la mise en place de modules spécifiques - et amélioré - par la régulation de modules existants - en proposant une offre de formation intégrant la question de la sensibilisation au genre.

21 L'introduction des nouveaux modules a également été saisie comme une opportunité pour faire évoluer le dispositif Did@cTIC vers une approche bilingue répondant ainsi mieux aux besoins du corps enseignant germanophone. En effet, l'Université de Fribourg étant une institution bilingue, il était important de répondre aux attentes du corps enseignant des deux parties linguistiques. Avant cette régulation, les enseignante-s germanophones désirant suivre une formation pédagogique dans leur langue devaient se déplacer à l'Université de Berne.

La démarche participative, basée sur l'observation, l'échange et le conseil individualisé est difficilement généralisable à l'ensemble du corps enseignant, car seule une petite minorité est sensibilisée de la sorte chaque année. Actuellement, la formation pédagogique du corps enseignant est facultative et encore peu valorisée. Une incitation plus marquée au niveau institutionnel pourrait avoir un impact. Une grille d'autoévaluation ou d'évaluation par les pairs pourrait alors constituer un outil intéressant pour favoriser la prise en compte de la dimension genre dans l'enseignement universitaire.

\subsection{Grille d'auto-évaluation}

A partir de l'analyse de la littérature existante et sur la base des premières expériences d'observation et de conseil aux enseignant-e-s intervenant dans les modules du dispoitif Did@cTIC, des critères de qualité pour un enseignement sensible au genre ont été définis. Ces critères ont été rassemblés et organisés pour former une grille d'autoévaluation ou d'évaluation par les pairs utile aux enseignant-e-s du supérieur. Cet outil d'évaluation est mis à disposition de tout enseignant-e intéressé-e par les questions genre sur le site du Centre de Didactique Universitaire ${ }^{1}$. Lors de son élaboration, diverses questions ont été soulevées telles que la validité de la grille, sa fonction (autoévaluation, co-évaluation) et sa comparaison avec d'autres grilles existantes du même type. Pour valider les questions de la grille, cette dernière a été testée auprès d'un échantillon d'enseignant-e-s. Un entretien auprès de chaque personne de cet échantillon a été effectué. Les questions posées ont été élaborées à partir des critères de validité proposés par Leclercq (2006): a) validité écologique; b) informative; c) conséquentielle; d) acceptabilité; et e) validité déontologique. Les résultats de cette démarche de validation mettent en évidence que la grille utilisée comme autoévaluation permet d'approfondir la réflexion sur son enseignement pour autant que les personnes soient déjà sensibilisées à la question du genre. Si tel n'est pas le cas, les enseignant-e-s ne perçoivent pas la pertinence des items de la grille. La nécessité d'une démarche de sensibilisation comme étape préliminaire renforce la pertinence de la mise en place de modules de formation tels que présentés au chapitre précédent. 


\section{Introduction de changements au niveau institutionnel}

L'ambition du projet e-qual ne se limitait pas à la mise en place de mesures individuelles pour l'intégration de la dimension genre dans la pratique enseignante. Elle visait également à développer un processus innovant de développement organisationnel en impliquant les divers acteurs clés de l'institution. Cette partie décrit les démarches et activités mises en place dans cette perspective.

\subsection{Mise en place d'un comité d'accompagnement}

Dès le début du projet, un comité d'accompagnement a été constitué, composé de deux expertes externes, deux expert-e-s internes de rang professoral (dont la directrice du Centre Didactique) et d'un représentant du Rectorat. Les autres membres du comité d'accompagnement étaient les responsables du Service de l'égalité et du Service d'évaluation et assurance qualité. Ce comité d'accompagnement a apporté un regard externe sur le projet e-qual. Les réunions du comité ont servi de plateforme d'échange permettant la confrontation des perspectives et besoins institutionnels à ceux du corps enseignant issus du projet e-qual, notamment par les communautés de pratique mises en place. Le comité d'accompagnement s'est rencontré à trois reprises durant le projet.

\subsection{Did@cTIC lunchs}

Dans la perspective de l'approche participative qui caractérise le projet e-qual, il s'agissait de pouvoir appuyer les mesures de sensibilisation à la dimension genre dans l'enseignement sur des expériences et préoccupations des membres du corps professoral exprimées dans le cadre d'échanges réflexifs au sein de communautés de pratique. Ces communautés réunissent un petit groupe de professeur-e-s et traitent d'une thématique en tenant compte des questions et/ou problèmes des participant-e-s, le but essentiel de chaque séance étant de permettre aux participant-e-s de trouver des pistes concrètes d'amélioration de leur pratique professionnelle. Ainsi, des communautés de pratique spécifiquement dédiées aux attentes des professeur-e-s, appelées Did@cTIC lunchs ont été mises en place suite à une analyse de besoins complétée par une série d'entretiens téléphoniques permettant de cibler les questions à aborder. Une liste des principales questions que se posent les professeur-e-s a ainsi été établie. Les thèmes suivants ont, par exemple, été évoqués :

1. Gestion de l'hétérogénéité des groupes. Comment prendre en compte les différences au niveau des attentes des étudiant-e-s? Comment considérer les différences au niveau des prérequis des étudiant-e-s?

2. Accompagnement et suivi individuel des étudiant-e-s ;

3. Suivi des évaluations. Comment tirer parti des évaluations d'enseignement institutionnalisées?

Quatre rencontres ont été organisées. Tous les professeur-e-s de l'Université de Fribourg ont été informés de la mise en place des Did@cTIC lunchs animés par la responsable du Centre de Didactique Universitaire. Selon la thématique traitée, un ou une expert-e de la question était également présent. Le nombre de participant-e-s a varié de trois à sept personnes. Les interactions ont été plus riches lors de la séance 
réunissant sept professeur-e-s. Cette communauté abordait la question «Créer et animer son équipe de travail». Lors de ce Did@cTIC lunch, une professeure de l'Université de Fribourg, spécialiste dans ce domaine, a joué le rôle d'experte.

Lors des Did@cTIC lunch, la dimension genre devait émerger des questions et problèmes relevés par les professeur-e-s eux-mêmes. L'objectif organisationnel de faire «remonter» les points de vue des enseignant-e-s en les relayant au sein du comité d'accompagnement et en tenant compte de ceux-ci dans le cadre de l'évaluation de l'enseignement n'a pas abouti. Différentes difficultés peuvent être évoquées pour expliquer cet état de fait comme la dynamique interne limitée des communautés de pratique. En effet, la difficulté de réunir un minimum de personnes a été réelle. Malgré une stratégie de communication et le souci de lever les freins potentiels à une telle participation (durée, moment choisi, confidentialité assurée...), le nombre de personnes présentes est resté très limité. Le rôle d'interface du comité entre le corps enseignant et le Rectorat était sans doute trop innovant par rapport au fonctionnement traditionnel. Ceci cumulé au nombre de rencontres limitées et discontinues explique la difficulté à mettre en œuvre ce rôle d'interface.

Malgré les difficultés rencontrées, les communautés ont été valorisées au niveau individuel. Les participant-e-s présent-e-s en ont tiré parti de diverses manières: apport d'informations, transfert dans sa propre situation professionnelle, validation de certaines pratiques, meilleure estimation de son autonomie dans son activité professionnelle.

\subsection{Le genre comme soutien au processus d'assurance qualité}

Dans le cadre du projet, une réflexion a été menée concernant la question des critères de qualité pouvant être utilisés comme indicateur d'un enseignement sensible au genre. Sur la base de la littérature existante concernant la question du genre et la gestion de la qualité de l'enseignement, un catalogue de critères a été établi. Ces critères ont été discutés au sein du groupe d'accompagnement. Ces échanges ont permis de réfléchir à la pertinence et faisabilité d'analyser la qualité de l'enseignement d'un point de vue genre dans les évaluations d'enseignement existantes effectuées au sein de l'Université de manière systématique. Trois options ont été considérées :

1. L'analyse de la sensibilité au genre par l'usage d'items spécifiques à cette question. Ce choix n'a pas été retenu car les expériences d'autres universités montrent clairement que les étudiant-e-s ne peuvent pas identifier ces aspects ;

2. Le repérage d'items du questionnaire officiel existant pour les regrouper en un indice de la sensibilité au genre des enseignant-e-s.

Cette option n'a pas été choisie car l'aspect genre ne peut pas être évalué uniquement sur la base des questions existantes. L'évaluation aurait été incomplète ;

3. L'analyse d'items existants en scindant les résultats selon le sexe des étudiant-e-s. Cette option a été valorisée par le groupe d'accompagnement.

31 Pour ce faire, un nouveau projet s'imposait. Nous avons soumis, dès lors, le projet equal+ visant à effectuer ces analyses. Dans ce projet, le travail sur la question du genre au niveau des évaluations d'enseignement permettra d'avoir une vue d'ensemble d'un groupe d'enseignant-e-s défini. Cette meilleure compréhension fournira des pistes visant à améliorer la sensibilisation au genre et permettra une approche plus systématique à portée institutionnelle. Par exemple, il deviendra possible de 
systématiser la sensibilisation au genre auprès des enseignant-e-s de l'université en leur fournissant des informations complémentaires lors de l'évaluation de leur enseignement. Le projet e-qual+ poursuit ainsi la réflexion et la démarche entreprise dans e-qual. Ce nouveau projet vise à ancrer la sensibilisation au genre auprès du corps enseignant de manière systématique. Le projet e-qual est, pour sa part, resté plus centré au niveau de changements individuels. La grille d'auto-évaluation (voir point 3.3) a été élaborée et diffusée pour stimuler cela.

\section{Conclusions et perspectives}

32 Le projet e-qual a permis d'accentuer la sensibilisation au genre du corps enseignant universitaire de Fribourg principalement par le développement du dispositif Did@cTIC et la mise à disposition d'un outil d'auto-évaluation. Cette première étape a atteint des changements essentiellement au niveau individuel. L'approche du développement organisationnel impliquant la participation et la coordination de trois services de l'institution universitaire a favorisé l'introduction de changements acceptés, soutenus et reconnus par chacun des services. Toutefois, si le but est de sensibiliser l'ensemble $\mathrm{du}$ corps enseignant à la question du genre dans l'enseignement, il sera nécessaire d'impliquer plus activement les enseignant-e-s dans cette démarche. Le projet e-qual+ va sensibiliser de manière plus large le corps enseignant en lui fournissant des informations sur la qualité de l'enseignement sous l'angle des différences de résultats selon le sexe. Cette approche sera complémentaire aux éléments mis en place dans le cadre d'e-qual. L'implication de représentant-e-s du Rectorat est une piste intéressante qui devra être poursuivie, car elle paraît indispensable dans une perspective de développement organisationnel. Le développement organisationnel participatif semble bien être une approche tout à fait pertinente pour favoriser des changements en profondeur et cohérents au sein d'une institution. Cependant, la démarche est complexe. Elle demande du temps et du tact. La généralisation d'une innovation telle que la prise en compte de la dimension de genre dans la qualité de l'enseignement par une majorité du corps enseignant reste délicate. Elle dépendra de l'engagement autant de la direction de l'institution, des milieux intermédiaires (décanats, départements) que des individus.

\section{BIBLIOGRAPHIE}

Deaux, K., \& Major, B. (1987). Putting gender into context : An interactive model of genderrelated behavior. Psychological Review, 94(3), 369-389.

Giauque, D., Barbey, V., \& Duc, N. (2008). Les leviers de la performance individuelle et collective dans les organisations publiques suisses : l'importance d'un pilotage participatif. Revue française d'administration publique, 4(128), 785-798. 
Gygax, P., Gabriel, U., Sarrasin, O., Oakhill, J., \& Garnham, A. (2008). Generically intended, but specifically interpreted : When beauticians, musicians, and mechanics are all men. Language and Cognitive Processes, 23(3), 464.

Leclercq, D. (2006). L'évolution des QCM. In G. Figari et L. Mottier-Lopez (Ed.), Recherches sur l'évaluation en éducation (pp. 139-146). Paris : L'Harmattan.

West, C., \& Zimmerman, D. H. (1987). Doing Gender. Gender and Society, 1(2), 125-151.

\section{NOTES}

1. Grille de critères pour l'évaluation de son enseignement: http://www.unifr.ch/didactic/ assets/files/didactic/Eval_cours_genre_fr.pdf

\section{RÉSUMÉS}

Les institutions d'enseignement supérieur sont nombreuses à développer l'égalité des chances. Dans ce texte, cette problématique est abordée sous un angle spécifique : le genre comme critère de qualité en enseignement supérieur. Le projet e-qual, mené à l'Université de Fribourg (Suisse), a impliqué divers services de l'institution tout en étant soutenu par le Rectorat. Le projet a développé la sensibilisation au genre du corps enseignant pour améliorer la qualité de l'enseignement en travaillant à un niveau individuel et institutionnel. L'élargissement de l'offre de formation Did@cTIC avec l'élaboration de modules liés à la question du genre ainsi que le développement d'une grille d'autoévaluation concernant la prise en compte du genre dans son enseignement sont des éléments concrets mis en place dans le cadre du projet. Au niveau institutionnel, les changements introduits ont été acceptés, soutenus et reconnus par les différents services concernés. La généralisation de la dimension genre en enseignement par une majorité du corps enseignant doit être poursuivie.

Many higher education institutions have been developing equal opportunities programmes. In this text, the topic addresses gender as a dimension of teaching effectiveness. The project e-qual carried out at the University of Fribourg (Switzerland) involved several departments and received the support of the University's Rector office. The project promotes teachers' awareness of gender issues in order to improve the quality of education working both at individual and institutional level. Designing new gender-related modules for the university's training programme Did@cTIC, and elaborating a self-evaluation questionnaire on gender issues in teaching are concrete elements implemented throughout the programme. At the institutional level, changes introduced have been accepted, recognized, and supported by the different departments involved in the project. Nevertheless extending gender issues in teaching for most of the academic staff requires further efforts. 
INDEX

Mots-clés : didactique universitaire, enseignement supérieur, genre, innovation, qualité enseignement

\section{AUTEURS}

\section{ANNICK ROSSIER}

Centre de Didactique Universitaire Boulevard de Pérolles 90

1700 Fribourg

annick.rossiermorel@unifr.ch

\section{JESSICA DEHLER}

Centre de Didactique Universitaire

Boulevard de Pérolles 90

1700 Fribourg

jessica.dehler@unifr.ch

\section{BERNADETTE CHARLIER}

Centre de Didactique Universitaire Boulevard de Pérolles 90

1700 Fribourg

bernadette.charlier@unifr.ch

HÉLÈNE FÜGER

Service de l'égalité

Avenue de l'Europe 20

1700 Fribourg

helene.fueger@unifr.ch 